\title{
Drilling the Data: Students Use Six Sigma DMAIC to Improve Dental Practice InVENTORY MANAgEMENT
}

\author{
Lisa M.Walters and Reneta Barneva
}

Lisa M. Walters, Assistant Professor

Reneta Barneva, Professor

The State University of New York-Fredonia

Fredonia, NY

Please send all correspondence regarding this paper to:

Lisa M. Walters Lisa.Walters@Fredonia.edu

Reneta Barneva Reneta.Barneva@Fredonia.edu

305 East Thompson Hall

The State University of NY-Fredonia

Fredonia, NY 14063

716-673-3505

\begin{abstract}
This work explains how data analytics within the framework of Six Sigma Quality, known as DMAIC (Define, Measure, Analyze, Improve, and Control), was used to diagnose and improve the inventory management process at a local dental practice which was operating at a loss. The analytics was done as part of a capstone
\end{abstract}


project. The students defined the problem using data mining, and further identified the scope of the problem using Critical-toQuality trees, Supply-Input- Process-Output-Customer (SIPOC) diagramming, and prioritizing the focus on the problem using a prioritization matrix. They further refined the business case and set specific goals for the improvement, in terms of revenue spent with regard to inventory and enumeration of expired materials. As a result, the dental practice got a report that allowed them to realize significant savings. Additionally, the students gained invaluable experience in taking a data-driven look at problems.

Keywords: Six sigma, inventory management, data analytics, business case

DOI: http://dx.doi.org/10.15239/j.brcacadje.2017.06.01.ja03

\section{INTRODUCTION}

Name any media outlet, and it's a safe bet that the spiraling costs of healthcare have been discussed, argued, solved, and resolved. When one thinks of healthcare costs, the predominant image may be one of hospitals, prescription drugs, and high-end medical devices. But what about dental medicine? Indeed, dentistry is a particular niche of medicine, with its own set of challenges when it comes to cost management. One source of costs for dental practices is the cost of inventory, the materials and supplies used in the procedures that ease pain, repair bites, and provide a winning smile.

This is a case study account of how 25 operations management (OM) students in the fall 2015 semester employed the project management framework of Lean Six Sigma (LSS), that is, Define-Measure-AnalyzeImprove-Control (DMAIC) (Brussee, 2004), through experiential learning in a 15-week operations management course in which these students evaluated and proposed improvements for the inventory management system of a local dental practice. Experiential learning has been found to engage students with the curriculum and to enhance their understanding 
of complex ideas (Papamarcos, 2002). Thus, this project was a perfect fit: enhance the learning of complex quality concepts of the students while assisting a community business with its own expense management and simultaneously educating them on the use of data to manage inventory.

\section{The Course Client: The Dental Practice}

The dental practice which participated in this course project has existed since 1972. Recognized as a "People's Choice Dental Practice" in its geographic area, this practice has enjoyed a history of financial success. To protect its market presence, the name of the practice will remain anonymous. This practice operates as a full-service, state-of-the art dental practice, providing general family dentistry and all phases of modern stomatology, including periodontal specialty services. The practice uses the latest technologies in preventive, restorative and cosmetic dentistry as a multi-service group practice. The practice consists of over 54 staff members, including doctors, hygienists, assistants, administrative and in-house laboratory staff.

This practice is located in an economically-challenged area, which suffered greatly in terms of employment as a result of the 2008 recession. Additionally, with a recently announced downsizing of a substantial employer, the practice has well-founded concerns about managing costs to ensure on-going business sustainability.

\section{The Lean Six Sigma DMAIC Framework}

LSS employs five essential phases to systematically effect an improvement in a process wherever such an improvement is needed (Furterer, 2013). These improvements may be in terms of efficiency as well as effectiveness, where efficiency is gained by the elimination or minimization of waste, and effectiveness is gained by achieving consistency in meeting identified targets with virtually zero defects. Each of the five phases of LSS has 
a specific goal, and each phase has different tools associated with it, some of which overlap phases. The Define phase has the aim to identify the project's scope, goal, and objectives as well as to understand the high-level process and that process's stakeholders, inputs, outputs, and functionality. The goal of the Measure Phase is to understand the process at a more detailed level, identify metrics to characterize the process, and measure it at a base level to serve as a basis for improvement. The Analyze Phase must aim to identify the influencers of the process's performance. The goal of the Improve Phase is to identify new process operating conditions and improvement recommendations. The Control Phase concerns establishing a plan to ensure that the gains in improvement are sustained.

While Lean Six Sigma had its first applications within manufacturing sectors, it is a methodology that may be applied to a service industry, enhancing productivity of operations and contributing to objectivity in results through the use of data driven analytics in lieu of guesswork. As a result, in healthcare, the tools of Lean Six Sigma can be implemented if practitioners understand the concepts of the methodology (Eckes, 2003). The understanding of LSS has an endless scope of possibilities. For example, there are institutions that have experienced more than a $50 \%$ improvement in patient satisfaction, reduced the length of service in the emergency department by $25 \%$, and gained more than $\$ 4$ million in the cost of quality, through the successful adoption of this procedure (Furterer, 2013).

The healthcare environment currently is an area, where the stakeholders in the practices must work together to optimize the performance of health delivery. Studies demonstrate an inflation of waste in health services (Goodwin \& Nick, 2005). Complicating this condition, many medical organizations do not consistently maintain comprehensive process documentation, making it very difficult to trace the sources of wastes and identifying subsequent amicable solutions to the same (Iyer \& Levin, 2006). 
Through LSS, not only is it possible to improve the quality of the health services in terms of delivery, but it is also possible to enhance the context of the healthcare delivery system, that is, the business aspects of the delivery system. Like most businesses, dental practices and other healthcare providers focus on return on investment (ROI). Careful analysis of problems through the adoption of LSS can make it easier for these providers to meet the ROI (George, 2003) and improvement targets they have established.

An additional benefit of LSS is that it is based fundamentally on customer needs, with resulting solutions verified by data to those customers' needs. As a result, solutions resulting from its application may be determined as more ethical when compared to solutions that result from hunches and guesswork (Furterer, 2013).

LSS then is ideal for all types of medical professionals, from dentists to nurses and middle level healthcare providers (Trsuko, 2007). Thus, LSS provides an excellent framework for this course project, allowing the students to apply LSS to the course client's (the dental practice) cost minimization problem, and in turn, teaching the course client of the benefits of data in managing its practice.

\section{Purpose and Research Questions}

The purpose of this study is to determine if business management students in an undergraduate OM course were able to apply the LSS concepts to provide a recommendation to the course client, an established dental practice that would achieve the client's identified business goal. An additional purpose was to determine whether this project would influence the approach the dental practice would take to future business problems. To this end, the following research questions guided the study:

Research Question 1: How likely are the recommendations developed by the students to result in the stated business goal of the course client? 


\section{The BRC Academy Journal of Education Vol. 6, No. 1}

Research Question 2: How likely will the results of this project be in influencing the approach the client takes on future business problems?

\section{Overview of THE OM COURSE}

The 15-week senior level OM course emphasizes the nature of OM and its inter-relationship with quality. This course is an applied class, as the students build on the concepts and themes learned earlier within their initial OM course with an emphasis on quality practices. The initial OM course provides the student with a conceptual understanding of the body of knowledge of OM. The general goals of this second OM course are to gain exposure to the various activities and decisions that involve an operations manager, learn about the trade-offs associated with these decisions, and acquire the conceptual and empirical tools for improving the performance of an organization.

In the fall 2015 course, 25 traditional, full-time college students (15 males and 10 females) were enrolled. These students majored in business administration, with a concentration in management. This course section met three times weekly for 50 minutes each meeting.

The overarching goal of the course was to apply the concepts of the QM tool of Lean Six Sigma (LSS) in assisting the dental practice described above to minimize cost expenditures; this problem was specifically related to the cost of its inventory management system. The course was based on learning centered on a conceptual understanding of QM practices and techniques as they apply to inventory management processes, with an emphasis on LSS and its systematic problem-solving model of Define-Measure-Analyze-Improve-Control (DMAIC). The students were randomly assigned into groups of equal size using the random group generator available in the learning management system. Between class meetings, the students applied the conceptual learning to the dental practice project, in terms of the DMAIC model, which resulted in deliverables to be presented at the next in-class meeting. For example, 
after an in-class discussion on Measure, the students determined their measurement plan for the dental practice's inventory management process and implemented that plan such that data were collected to describe the process behavior. At the next class, time was spent on evaluating that data as a class and then using that data to segue to the next phase of DMAIC, i.e., Analyze. During discussions with the professor, students would be individually called upon to provide an in-depth update regarding the project. The professor would randomly select students within the groups to provide this update. The intent of this questioning was to assure the professor that individuals were contributing to the group, as opposed to allowing others to do the work and simply receive the same grade. If an individual could not contribute to this update, the professor would withhold 10 points from that student's score for the project. Additionally, that same student would be questioned more frequently, thereby maintaining a higher risk of losing more substantial points. This method appeared to be rather effective, as students maintained meticulous notes and were consistently able to provide the professor with in-depth information when randomly selected. Further, no groups complained to the professor about any one group member throughout the progression of the project. Additionally, students individually completed quizzes and essays concerning Six Sigma concepts to determine differences in performance among the students with regard to content understanding and project participation.

Toward the end of the semester, a report was prepared by the students that described the project and included all phases of the DMAIC model. It also included a formal presentation of the project to the course client.

\section{Overview of the LSS Course Project}

To begin the project, students were introduced to the LSS process through in-class lectures, in-class exercises, and required readings. In this way, the students were given an overview of the DMAIC model, which set the stage for the more detailed DMAIC work on the dental practice's 


\section{The BRC Academy Journal of Education Vol. 6, No. 1}

inventory management business problem. These tasks took several weeks, and when a foundation of LSS was in place, representatives of the dental practice visited the class to provide information on the current inventory management process. Because the students did not directly access any patient information but rather focused on anonymous data sets, health care privacy was not a concern in this particular case. However, in general, such concerns should be taken into consideration in health data analytics. The client class visit began the Define Phase of the project.

\section{Define}

The dental practice representatives provided both qualitative and quantitative information to the students. Quantitatively, the practice shared the last year of inventory materials purchases, including quantity and costs. From the data, it was determined that $10 \%$ of revenue is expended on inventory, where inventory was defined as the expendable materials used in specific dental procedures, such as crown prep, restorations, cleanings, etc. Additionally, data provided to the students by the practice originating from a large dental inventory supplier indicated that the percentage of revenue expended to inventory should be close to $5 \%$. Additionally, the dental practice identified about $\$ 5000$ worth of expired or out-of-date inventory in the inventory storage areas.

Qualitatively, they described a high-level process flow of the current state of the inventory ordering process. For each process step, the suppliers of each input to that process step were identified, as well as the outputs and the customers of those. This information was used in the Suppliers-Inputs-Process-Outputs-Customers (SIPOC) diagramming tool to establish the boundaries of the project. (See Fig. 1)

This diagram illustrates that the focus on the inventory management process is perceived as existing within the inventory ordering process. But which aspect of that process?

To begin answering this question, the students interviewed the practice representatives to describe their particular needs in terms of the inventory 
management system. These requirements were considered as the Voice of the Customer ( $\mathrm{VoC}$ ) for the inventory management process. They were organized as a Critical-To-Quality (CtQ) diagram. (See Fig. 2)

The CtQ Diagram identifies not only the requirements of the customers of the inventory management system (the practitioners), but also more specifically, how these requirements can be translated into measurable terms. Its construction begins with identifying broader needs of the customers and then drilling into those needs to better operationally define what the needs mean. Finally, a measure is associated with the requirements, which will be valuable again later during the Control Phase of DMAIC.

The high-level inventory management process was mapped to the VoC requirements using the primary matrix of the Quality Function Deployment (QFD) tool, which facilitates understanding of the requirements that please a customer and the process attributes that are essential to those requirements. (See Fig. 3)

This matrix is constructed by listing the measures from the $\mathrm{Ct} Q$ diagram in the first column, with the larger process steps from the SIPOC listed as the first row of the matrix. Weights are assigned for the CtQ measures, as defined by the customer (the practice representatives). In this case, if the measure was considered very important, it was weighted a 5 . If it was of moderate importance, it was weighted a 3 . If it was not of any great importance, it was rated a 1.

The relationship between the measures and the process steps from the SIPOC were then determined and ranked, using a $1-9$ scale, with 1 being little to no relationship, and 9 translating into a very strong relationship. This relationship rating was done with the practice representations. Each relationship rating was multiplied by the weight of the customer's measure, providing a weighted relationship rating. These weighted ratings were totaled for each column, and divided by the total weight of the customer measures, providing a weighted average. The process step with the highest weighted average is the area of focus for the 
process, thereby providing the scope of the problem. Hence, this tool demonstrated the need to improve the process of identifying the amount of inventory to order.

As a result of this phase, the problem was defined as: the process to identify needed inventory is not effective to ensure that expenditures on inventory as a function of revenue do not exceed 5\%. Currently the practice spends $10 \%$ of revenue on inventory. Additionally, expired products have been identified in available inventory. The goal is to reduce the percentage of revenue spent on inventory to 5\% and reduce the amount of expired inventory to zero.

\section{Measure}

The Measure Phase seeks to characterize the process as it exists prior to the improvement. Qualitatively, to understand the way the dental practice identified how much inventory it needed, the inventory clerk was interviewed and the process mapped. From the interview, it was determined that no formalized process existed, and the inventory clerk essentially ordered inventory by intuition based on historical usage. Additionally, the clerk had no formal training in inventory management, nor did the clerk have a designee when she was unavailable.

Quantitatively, an ImR chart was constructed to determine if the inventory ordering process capability in terms of the number of times a month orders were placed. An ImR chart is a graphical representation of process performance comprised of two separate charts, with the $I$ chart displaying the individual value of each measurement with the corresponding average of values, and $m R$ chart representing the variation of each measurement in terms of the preceding point. The $\operatorname{ImR}$ chart is used as part of statistical process control (SPC) to monitor processes and detect any special causes which may signal the process could move out of predictable movement ("out of control"). It also provides, as part of the I chart, the upper control limit (UCL) and the lower control limit (LCL), representing the expected high and low values of the process movement. 
The information in terms of the number of times a month an order was placed was charted, as the dental practice owners desired an order to be placed only several times per month, to minimize ordering costs and time spent in ordering. (See Fig. 4.)

The ImR chart illustrates that the number of orders placed per month can range from a low of about 14 orders to a high of about 33 orders, with an average of about 23 . These values are clearly much higher than several times per month desired by the practice. In September, an out-ofcontrol data point indicates a trend below the lower control limit, which coincides with the actions taken by the dental practice management to limit orders beginning in May. In May, the practice began to realize the amount of money being spent on inventory; as a result, the practice management directed the inventory clerk not to order any more inventory until the dentists gave the authorization to order based on inventory depletion. This action resulted in less monthly orders; however, the need to order was reactive and not based on any systematic method of inventory control.

\section{Analyze}

The analysis phase seeks to establish the data gathered in the measure phase to ascertain the most basic of reasons for the stated problem. The tool used for this problem analysis was the fishbone diagram, which is used to align possible causes of a problem and then attempt to drill into those possible causes to determine a root cause. Six dimensions of process inputs were evaluated as part of the fish boning exercise: human (man), method, supplies (materials), measurement, equipment (machine), and environment. (See Fig. 5.)

A number of possible causes were identified as part of this analysis. Although the equipment bone was essentially devoid of data, some of the other bones exhibited an area of commonality: a lack of written procedure, which would include critical information for the ordering 
process, such as when an order should be placed, as defined by standard stock levels and reorder points (ROPs).

\section{Improve}

To improve the process, standard inventory levels and reorder points for critical materials for six key dental processes were established. These procedures were: hygiene, amalgam restoration, composite restoration, crown preparation, endodontic surgery, and post and core.

For each dental procedure and with the input of the dentists, a bill of materials (BOM) was generated. These BOMs were used chairside for a period of several weeks to capture average use of each material per procedure. Times series forecasts were conducted for each procedure. The forecasts were for a 12-month horizon. The standard stock levels were calculated as the product of the average use of the material per procedure and the forecasted average of procedure type per month, providing an exploded BOM.

The standard stock levels were used to determine the reorder points for each material according to the formula:

(Demand for the material per year/Number of working days per year) $)^{*}$ Lead time.

Demand for the material per year was the standard stock level for the year. The practice operates for 250 days per year. The lead time was established as three days and represents the time elapsed from the time an order is placed until the time it is received.

To implement the reorder points, the storage areas of the dental practice were subjected to the lean tool of 5-S (sort, set in order, shine, standardize, and sustain). An intern was selected to assist in this endeavor. First, all inventory areas were evaluated and items not necessary to the dental procedures were removed and put into long term storage in the basement. The remaining inventory was organized with regard to frequency of use and by procedure. All shelving and inventory locations were labeled with 
item type and re-order point for that item. This labeling assists in keeping the areas orderly and consistent. Lastly, a procedure was developed describing how inventory items would be ordered going forward, based on the reorder points. This procedure constituted the standard work of the inventory management system.

\section{Control}

To ensure the sustainability of this improvement, all staff members were trained on the new procedure, and job descriptions were updated reflecting accountability for compliance to the procedures. One aspect of the new procedure requires the inventory clerk to physically evaluate the inventory areas to ensure that all items remain set in order, and no expired inventory exists. Additionally, a visual graphic demonstrating the cost of inventory as a function of revenue was posted in the staff break area so that cost savings could be easily viewed by staff. This visual control had the additional benefit of allowing staff to understand the extent of expenditures required by the practice, resulting in an increase in staff commitment toward this cost-saving effort as evidenced by staff offering further cost-savings ideas for consideration by the practice, including and beyond the inventory system.

\section{Methodology}

To answer the research questions, a mixed-methods approach was taken. To answer Research Question 1, quantitative data were evaluated. To answer Research Question 2, qualitative data were evaluated. Each research question was evaluated as described below.

Research Question 1: How likely are the recommendations developed by the students to result in the stated business goal of the course client?

To answer this research question, the monthly revenue of the dental practice was determined. Additionally, for those same months, the cost of inventory was calculated. If the percent of revenue spent on inventory 
over those months appear to be moving in a downward direction, it appears likely the improvement would result in reducing inventory as function of revenue to approximately $5 \%$, the stated business goal of the practice. Additionally, no expired inventory should be identified in the storage areas.

Research Question 2: How likely will the results of this project be in influencing the approach the client takes on future business problems?

To answer this research question, the dentists would be interviewed by the student-researchers in a non-structured setting to answer the following: Given the outcome of this project, what surprised you and what would you, as a practice, do differently, if anything? If the interview yielded a future orientation toward data-driven decision-making as well as employee engagement in solving practice issues, this research question would be considered to be answered in the affirmative sense.

\section{Results}

The data were collected two months after the implementation of the improvement. Each question will be considered separately below.

Research Question 1: How likely are the recommendations developed by the students to result in the stated business goal of the course client?

The monthly revenue of the dental practice was determined for the two months immediately after the implementation of the improvement. For the first month, inventory costs represented $8 \%$ of revenue, while in the second month, inventory costs represent $7 \%$ of revenue. During continued evaluation, the inventory costs as a function of revenue were determined as $5 \%$. This percentage has been maintained for a period of seven months, the latest figures available to this research team. This percentage represents $\$ 250,000$ returned to the practice. Additionally, no expired inventory was located in the inventory storage areas. 
Research Question 2: How likely will the results of this project be in influencing the approach the client takes on future business problems?

The unstructured interview was conducted by the research team. The dentists were asked: "Given the outcome of this project, what surprised you and what would you, as a practice, do differently, if anything?" The dentists indicated they were surprised by the systematic nature of the problem-solving effort, which they believed assisted them in gaining staff buy-in to the problem-solving effort. For example, because the problem was approached using data and not using personal attacks for failure to save money on inventory, staff were more likely to assist the practice in achieving the goal, offering suggestions and ideas for even more improvements. The dentists were also surprised by the power of the visual graphic detailing inventory expenses, noting this graphic generated quite a bit of discussion and helped staff understand the magnitude of necessary expenditures to sustain the practice. Staff members generally commented that "we didn't know how much money we spent on inventory!" The dentists also found that standard work and training in standard work with regard to inventory management assisted them in getting the inventory system up and running in a shorter period of time. Because of the standard work and training, everyone was clear on his or her role within the inventory management system, while embedding these roles within the job descriptions assisted in facilitating accountability for compliance with the inventory management system.

Going forward, the dentists indicated they would engage their staff more readily in the problem-solving effort, and make the expectations for problem solving solutions transparent and formal. Lastly, they indicated they would try to use data as much as possible in problem solving, because they agreed the focus on the data helped make the problemsolving effort objective and not staff focused. 
72 The BRC Academy Journal of Education Vol. 6, No. 1

\section{Discussion}

The results of the study indicate that the Lean Six Sigma methodology is valuable not only to traditional manufacturing, but also to the service industries, such as a dental practice. This finding is consistent with that of Trsuko (2007), who indicated that indeed this methodology can benefit healthcare endeavors. The project to date has eliminated waste as readily evidenced in the lack of expired materials noted, as well as the amount of revenue spent on inventory purchases, consistent with the findings of George (2013) and Furterer (2013), who both noted that LSS can improve a firm's return on investment (ROI) and facilitate cost savings.

Additionally, this case study appears to have had the impact of influencing the dental practice to employ more data-driven solutions in the future, with an emphasis on the process that leads to the issues, rather than focusing on staff behaviors. This focus provides a more ethical framework of problem-solving, as noted by Furterer (2013), as staff are not immediately blamed for the problem at hand, and are, instead, considered partners in solving the issues, which may result in more robust and sustainable outcomes for all stakeholders, including patients. By continually employing the tools, the practice will undoubtedly become more proficient in the applications of this methodology, supporting the work of Eckes (2003).

\section{Limitations ANd Further StUdy}

This study was limited by several factors. The first factor is the amount of time available to complete the project. Because this project spanned only one academic semester, the amount of data collected during the measurement phase was limited. More data collected during that time frame may provide more robust reorder points; because the improvement is early in its implementation it is difficult to be entirely sure that the ROPs determined will be stable over the forecast period of one year. An additional consideration resulting from the time available for the project 
completion is the number of dental procedures considered. Six dental procedures were selected by the practice for analysis by the students; these were selected based on their frequency of occurrence. However, other dental procedures do exist which may influence the amount of revenue expended on inventory. To counter these limitations, it would be helpful to run the project again, perhaps over two semesters, to collect more measurement data and to also include all dental procedures.

A second limitation of the study was the current economic condition of the geographic location in which the practice is located. Currently, the major employer of that area is undergoing a relocation of much of its work to another manufacturing plant. As a result, dental insurances of those employees will no longer be available, many employees may need to leave the area, and economic instability will more than likely be introduced into the area. Thus, the forecasts of the dental procedures may not be stable, thereby influencing the integrity of the stock quantities. As a result, it may take longer to reach the ROPs, resulting in expired materials. Thus, it is critical that the practice monitor expired materials as part of its control phase.

\section{REFERENCES}

Brussee, W. (2004). Statistics for Six Sigma Made Easy. McGraw-Hill Companies.

Eckes, G. (2003). Six Sigma for Everyone. Hoboken, New Jersey: John Wiley \& Sons, Inc.

Furterer, S. (2013). Lean Six Sigma Case Studies in the Healthcare Enterprise. Marietta, GA: Springer.

George, M. (2003). Lean Six Sigma for Service. McGraw-Hill Companies.

Goodwin, D., \& Nick, G. (2005). Managing Health Services. Berkshire: Open University Press.

Harry, M., Mann, P., \& DeHodgins, O. (2011). Practitioner's Guide to Statistics and Lean Six Sigma for Process Improvements. Wiley. 
74 The BRC Academy Journal of Education Vol. 6, No. 1

Iyer, W., \& Levin, B. (2006). Medical Legal Aspects of Medical Records. Lawyers \& Judges Publishing Company, Inc.

Papamarcos, S. D. (2002, Spring). The "next wave" in service-learning: Integrative, team-based engagements with structural objectives. Review of Business, 31-38.

Trsuko, H. (2007). Improving Healthcare Quality and Cost with Six Sigma. Pearson Education.

\section{Citation Information}

Walters, Lisa M., and Reneta Barneva. "Drilling the Data: Students Use Six Sigma DMAIC to Improve Dental Practice Inventory Management ." The BRC Academy fournal of Education 6, no. 1 (2017): 57-74. http:// dx.doi.org/10.15239/j.brcacadje.2017.06.01.ja03

\section{Web APPE ndix}

A web appendix for this paper is available at:

http://dx.doi.org/10.15239/j.brcacadje.2017.06.01.wa03 